\title{
Massive Oe/Be stars at low metallicity: candidate progenitors of long GRBs?
}

\author{
Christophe Martayan ${ }^{1,2}$, Dietrich Baade ${ }^{3}$ Juan Zorec ${ }^{4}$, Yves Frémat ${ }^{5}$, \\ Juan Fabregat ${ }^{6}$ and Sylvia Ekström ${ }^{7}$ \\ ${ }^{1}$ ESO Chile; ${ }^{2}$ GEPI-Observatoire de Meudon, France; ${ }^{3}$ ESO Germany; \\ ${ }^{4}$ Instritut d'Astrophysique de Paris, France; ${ }^{5}$ Royal Observatory of Belgium, Brussels, Belgium; \\ ${ }^{6}$ Valencia University, Spain; ${ }^{7}$ Geneva Observatory, Switzerland
}

\begin{abstract}
At low metallicity B-type stars rotate faster than at higher metallicity, typically in the SMC. As a consequence, a larger number of fast rotators is expected in the SMC than in the Galaxy, in particular more Be/Oe stars. With the ESO-WFI in its slitless mode, we examined the SMC open clusters and found an occurence of Be stars 3 to 5 times larger than in the Galaxy. The evolution of the angular rotational velocity seems to be the main key on the understanding of the specific behaviour and stellar evolution of such stars at different metallicities. With the results of this WFI study and using observational clues on the SMC WR stars and massive stars, as well as the theoretical indications of long gamma-ray burst progenitors, we identify the low metallicity massive Be and Oe stars as potential LGRB progenitors. Therefore the expected rates and numbers of LGRB are calculated and compared to the observed ones, leading to a good probability that low metallicity Be/Oe stars are actually LGRB progenitors.
\end{abstract}

Keywords. gamma rays: observations, stars: supernovae: general, stars: rotation, Magellanic Clouds

\section{Long GRBs and the collapsar model}

In the collapsar model for Ib/c supernovae (Woosley 1993), matter with high specific angular momentum is accreted by the already-formed black hole with a short delay. The intermediate disk structure gives rise to the formation of relativistic jets emitting the so-called long gamma-ray bursts (GRBs). The gamma-ray radiation is strongly relativistically beamed with opening angles of order 10 degrees.

In order for a rapidly rotating core to be present, quasi-homogeneous chemical evolution is essential. Stellar evolution models show that, in stars with very high initial rotation rates, mixing is faster as chemical gradients are built up by nuclear burning (for more details see Yoon \& Langer 2005).

The angular momentum still available at this late phase of stellar evolution is the larger, the less mass, and therefore, angular momentum, the progenitor star has lost during its lifetime. Since radiatively driven mass loss from massive stars decreases with metallicity, this has led to the notion that low-metallicity star-forming regions should be preferred hosts of GRBs. In fact, observations are beginning to support this picture (e.g., Modjaz et al. 2008). Moreover, the GRB rate per unit mass increases with redshift $z$. But it is not clear whether this requires any explanation other than the general increase in star formation with redshift (up to $\mathrm{z} \sim 2$ ).

\section{Oe/Be stars}

In the Milky Way, the near-main sequence stars with the highest rotation velocities are Oe/Be stars. Moreover, many of them rotate at $\sim 90 \%$ of the critical rate. Two arguments 
predict a larger fraction of nearly critically rotating massive stars in low-metallicity environments: (i) The mass (and angular momentum) loss rates are lower and (ii), all else being equal, the radii are smaller. Various comparisons of open clusters in the Magellanic Clouds and the Galaxy have concluded (cf. Martayan, Baade \& Fabregat 2010) that the abundance of Oe/Be stars increases with decreasing metallicity $Z$. If one keeps in mind that rapid rotation only is a necessary, but not also sufficient, condition for a rapidly rotating $\mathrm{O} / \mathrm{B}$ star to become an $\mathrm{Oe} / \mathrm{Be}$ star, this can pass as a confirmation of the expectation. In any event, because of their emission lines, Oe/Be stars probably are more reliable tracers of a population of rapidly rotating OB stars than broad photospheric lines would be. Therefore, Oe/Be stars more massive than $\sim 18 M_{\odot}$ (metallicity dependent; Yoon, Langer \& Norman 2006) qualify as candidates, also at low $Z$. Note that, perhaps, Oe/Be stars do not quite reach the theoretical upper mass limit for the progenitors of GRBs (Yoon, Langer \& Norman 2006).

\section{GRBs and Oe/Be stars in a synopsis}

The strong relativistic beaming makes GRBs observable out to extreme redshifts. But it also implies that much fewer nearby GRBs are detected. Therefore, while it is still relatively straightforward to determine the number of Oe/Be stars per SMC mass and also the space density of such environments as a function of redshift can be estimated, statistics with just a few dozen entries (GRBs) pose serious problems. Nevertheless, using a distribution of beam angles determined from observations, 3-6 long GRBs are predicted at $z \leqslant 0.2$ per 11-year period while the observed number is 8 (cf. Martayan et al. 2010). Since the agreement is better than $2 \sigma$, there is no need to fine tune adjustment parameters such as the range in spectral type, from which GRB progenitors are drawn, or the number, type, and star-formation level of the assumed host galaxies. But the excellent agreement could still be coincidental.

\section{Discussion}

In addition to the challenges already mentioned, a number of other questions still warrant further examination:

- Do Oe/Be stars really preserve their large intial angular momentum throughout their evolution? After all, they regularly eject rotationally supported disks. This behavior is not observed in On/Bn stars, i.e. rapidly rotating O/B stars without emission lines.

- Are there other differences in the evolution of Be and Bn stars?

- Is the SMC a good proxy for GRB-forming environments?

- How incomplete are the observations, especially of GRBs?

- Can candidate progenitors other than Oe/Be stars reach the GRB state along different evolutionary paths?

\section{References}

Martayan, C., Baade, D., \& Fabregat, J. 2010, A\&A, 509, A11

Martayan, C., Zorec, J., Frémat, Y., \& Ekström, S. 2010, A\& A, 516, A103

Modjaz, M., Kewley, L., Kirshner, R. P., Stanek, K. Z. et al. 2008, AJ, 135, 1136

Woosley, S. E. 1993, ApJ, 405, 273

Yoon, S.-C. \& Langer, N. 2005, A\&A, 443, 643

Yoon, S.-C., Langer, N., \& Norman, C. 2006, A\&A, 460, 199 